\title{
Involvement of miRNAs in equine follicle development
}

\author{
S N Schauer, S D Sontakke, E D Watson ${ }^{1}, \mathrm{C}$ L Esteves ${ }^{2}$ and F X Donadeu \\ The Roslin Institute and Royal (Dick) School of Veterinary Studies, University of Edinburgh, Easter Bush, Edinburgh, \\ Midlothian EH25 9RG, UK, ${ }^{1}$ Ross University School of Veterinary Medicine, PO Box 334, Bassterre, Saint Kitts, \\ West Indies and ${ }^{2}$ Endocrinology Unit, BHF Centre for Cardiovascular Sciences, University of Edinburgh, Edinburgh \\ EH16 4TJ, UK
}

Correspondence should be addressed to FX Donadeu; Email: xavier.donadeu@roslin.ed.ac.uk

\begin{abstract}
Previous evidence from in vitro studies suggests specific roles for a subset of miRNAs, including miR-21, miR-23a, miR-145, miR-503, miR-224, miR-383, miR-378, miR-132, and miR-212, in regulating ovarian follicle development. The objective of this study was to determine changes in the levels of these miRNAs in relation to follicle selection, maturation, and ovulation in the monovular equine ovary. In Experiment 1, follicular fluid was aspirated during ovulatory cycles from the dominant (DO) and largest subordinate (S) follicles of an ovulatory wave and the dominant (DA) follicle of a mid-cycle anovulatory wave $(n=6$ mares). Follicular fluid levels of progesterone and estradiol were lower $(\boldsymbol{P}<\mathbf{0 . 0 1})$ in $\mathrm{S}$ follicles than in DO follicles, whereas mean levels of IGF1 were lower $(P<0.01)$ in $\mathrm{S}$ and DA follicles than in DO follicles. Relative to DO and DA follicles, $S$ follicles had higher $(P \leq 0.01)$ follicular fluid levels of $\mathrm{miR}-145$ and $\mathrm{miR}-378$. In Experiment 2, follicular fluid and granulosa cells were aspirated from dominant follicles before (DO) and $24 \mathrm{~h} \mathrm{after} \mathrm{(L)} \mathrm{administration} \mathrm{of} \mathrm{an}$ ovulatory dose of hCG ( $n=5$ mares/group). Relative to DO follicles, $L$ follicles had higher follicular fluid levels of progesterone $(P=0.05)$ and lower granulosa cell levels of CYP19A1 and LHCGR $(P<0.005)$. Levels of miR-21, miR-132, miR-212, and miR-224 were increased $(P<0.05)$ in $L$ follicles; this was associated with reduced expression of the putative miRNA targets, PTEN, RASA1, and SMAD4. These novel results may indicate a physiological involvement of miR-21, miR-145, miR-224, miR-378, miR-132, and miR-212 in the regulation of cell survival, steroidogenesis, and differentiation during follicle selection and ovulation in the monovular ovary.

Reproduction (2013) 146 273-282
\end{abstract}

\section{Introduction}

Posttranscriptional gene regulation by microRNAs (miRNAs) plays a key generic role during tissue development and differentiation (reviewed in Sayed \& Abdellatif (2011)). Currently, more than 2000 different mature miRNAs are registered in humans (MiRBase 19.0), with relatively lower numbers identified in other species (360 miRNAs in the horse). Given the highly dynamic nature of the adult ovary, it is not surprising that several hundred miRNA sequences have been identified in this organ in different species (Mishima et al. 2008, Hossain et al. 2009, Li et al. 2011, McBride et al. 2012). Furthermore, the expression of a subset of miRNAs has been shown to vary during follicle/luteal development (Fiedler et al. 2008, Lin et al. 2012, McBride et al. 2012). Yet, compared with other body tissues, very little is known about the physiological roles of miRNAs during follicular development. Effects on granulosa cell function and/or gene expression, largely in mice, have been reported for a handful of miRNAs (Carletti et al. 2010, Lei et al. 2010, Yao et al. 2010, Xu et al. 2011, Lin et al. 2012, Yan et al. 2012, Yang et al. 2012, Yin et al. 2012), and these include effects on cell survival (miR-21 and
miR-23a), proliferation (miR-145, miR-503, and miR224), estradiol production (miR-224, miR-383, and miR378), and terminal differentiation (miR-132 and miR-212) of cultured cells (reviewed in Donadeu et al. (2012)). In contrast, an involvement in folliculogenesis in vivo has only been shown for one miRNA; specifically, Carletti et al. (2010) showed that miR-21 acted as a prosurvival factor in murine granulosa cells during hCGinduced ovulation. Importantly, considering differences in ovarian physiology between species, it is not known whether the roles of follicular miRNAs in rodent models are conserved in monovular species such as humans and horses. In that regard, the mare provides a unique model for studying follicular physiology in the monovular ovary due to i) the large size and easy experimental accessibility of ovarian follicles, which allow specific stages of follicle development to be precisely monitored in vivo to an extent which is not feasible in other species (Donadeu \& Ginther 2002a, 2002b), and ii) the reported similarities in follicular dynamics between horses and humans (Ginther et al. 2004, 2005, Carnevale 2008). In a recent quantitative PCR (qPCR) screen, very similar miRNA populations were found in antral fluid 
and granulosa cell compartments of equine follicles (da Silveira et al. 2012), suggesting that antral fluid samples may be useful to monitor follicular miRNA status.

The objective of this study was to determine changes in the levels of miRNAs occuring during follicular development in the monovular ovary. The study focused on a subset of miRNAs for which a functional role in regulating follicular cells has been proposed, mostly in mice, namely, miR-21, miR-23a, miR-145, miR-503, miR-224, miR-383 miR-378, miR-132, and miR-212 (see above). This was done by determining the changes in the levels of miRNAs in individual follicles at well-defined developmental stages encompassing follicular selection, maturation, and ovulation in mares. The overall hypothesis was that the physiological changes in miRNA levels across follicle stages would be in each case consistent with follicular roles proposed in the earlier in vitro studies.

\section{Materials and methods}

\section{Experimental animals and general procedures}

Welsh cross pony mares, 3-14 years of age, with body weights between 250 and $425 \mathrm{~kg}$ and a history of good reproductive health, were kept under natural light with access to grass and haylage, water, and to mineral salts ad libitium. Two experiments were carried out during the natural breeding season (May to October) in the northern hemisphere $\left(55^{\circ} \mathrm{N}\right.$, Edinburgh, UK) under the UK Home Office Animals (Scientific Procedures) Act 1986 with approval by the Ethical Review Committee, University of Edinburgh.

Ovaries were monitored by trans-rectal ultrasonography using a 7.5 MHz transducer on a DP-6600 Vet Digital Ultrasonic Diagnostic Imaging System (BCF Technology, Livingston, UK). Follicle diameters were determined by measuring follicle size in two perpendicular planes and taking the average of length and width as the actual diameter. Ovulation was established by the disappearance of a preovulatory follicle and the presence of an echoic corpus luteum.

Follicular aspiration was performed by trans-vaginal ultrasound-guided follicle puncture using a 17 gauge, $55 \mathrm{~cm}$ long ovum pickup needle (Popper \& Sons, Inc., New Hyde Park, NY, USA) and an Aloka 500V ultrasound scanner (BCF Technology, Rochester, MN, USA) equipped with a $5 \mathrm{mHz}$ curved array transducer, as described in Doyle et al. (2008). Antral fluid was completely aspirated from each follicle and aspirates were centrifuged at $700 \mathrm{~g}$ for $10 \mathrm{~min}$ to separate granulosa cell pellets from fluid supernatant. Follicular fluid was stored at $-20{ }^{\circ} \mathrm{C}$ until analyses. Cell pellets were re-suspended with $1 \mathrm{ml}$ red blood cell lysis buffer $\left(10 \mathrm{mM} \mathrm{KHCO}_{3}, 150 \mathrm{mM}\right.$ $\mathrm{NH}_{4} \mathrm{Cl}$, and $0.1 \mathrm{mM}$ EDTA, $\mathrm{pH}$ 8.0) for $1 \mathrm{~min}$ to remove residual blood contamination and then stored in $500 \mu \mathrm{l}$ RNABee (AMS Biotechnology, Abingdon, UK) at $-80^{\circ} \mathrm{C}$. Pellets that were grossly contaminated with blood were discarded.

\section{Experiment 1}

Samples were collected during the course of an earlier study (Schauer et al. 2013). Follicles and corpora lutea were monitored daily by ultrasonography beginning 8 days after ovulation in each of six mares; this was done to identify naturally occurring ovulatory and anovulatory waves developing during the follicular phase and the luteal phase, respectively, of an estrous cycle. In each mare, the dominant and largest subordinate follicles of an ovulatory wave were aspirated once the dominant follicle reached a preovulatory diameter of $>32 \mathrm{~mm}$. In addition, an anovulatory wave was identified by the presence of one or more actively growing $>29 \mathrm{~mm}$ follicles (Donadeu \& Pedersen 2008) during midcycle (days 9-12 after ovulation) and the largest follicle was aspirated. Follicular aspirates were in all cases processed for later analyses as described earlier.

\section{Experiment 2}

Six mares were assigned to each of the two experimental groups using a randomized crossover design so that each mare was assigned to each group once. Each mare was allowed one full ovulatory cycle between the two experimental periods. Beginning 15 days after ovulation, follicle growth was monitored daily. When the largest follicle of a natural ovulatory wave reached a diameter $>32 \mathrm{~mm}$, the follicle was either aspirated or an ovulatory dose of hCG was administered (3000 UI i.v.) and the follicle aspirated $24 \mathrm{~h}$ later. Most mares with a preovulatory follicle typically ovulate between 36 and $48 \mathrm{~h}$ following administration of hCG (Gastal et al. 2006). Follicle aspirates were processed as described earlier.

During both experiments, after each follicular aspiration ovaries continued to be monitored regularly to ensure that mares continued to ovulate normally. This was done to prevent mares entering seasonal anestrus from being included in the study.

\section{ELISA and RIA analyses}

Commercial ELISA kits were used to determine concentrations of estradiol (DRG Instruments GmbH, Marburg, Germany), $\mathrm{PGE}_{2}$ (GE Healthcare, Little Chalfont, UK), and IGF1 (Diagnostic Systems Laboratories, Inc., Webster, TX, USA), as described in Schauer et al. (2013). Intra-assay coefficients of variation (CV) were $9.8,7.9$, and $9.9 \%$ and assay sensitivities were $5.2,40$, and $15 \mathrm{pg} / \mathrm{ml}$ respectively. Progesterone levels in follicular fluid were measured with a Coat-a-Count RIA kit (Siemens Healthcare Diagnostics, Inc. Tarrytown, NY, USA), as described. Intra-assay CV was $4.3 \%$ and assay sensitivity was $0.01 \mathrm{ng} / \mathrm{ml}$. All assays were validated in our laboratory by showing parallelism between serial sample dilutions and the provided assay standard curve. Sensitivity was in all cases calculated by subtracting two S.D. from the mean optical density or counts per minute value at maximum percentage binding.

\section{Gene expression analyses}

Total RNA was extracted from granulosa cell pellets using RNA-Bee reagent according to manufacturer's instructions and was quantified using a ND-1000 spectrophotometer (NanoDrop Technologies, Wilmington, DE, USA). Follicular fluid samples were spun at $10000 \mathrm{~g}$ for $10 \mathrm{~min}$ at $4{ }^{\circ} \mathrm{C}$ to remove cell debris and $200 \mu \mathrm{l}$ supernatant were placed in Qiazol buffer 
(Qiagen) containing MS2 carrier RNA (Roche Diagnostics Ltd.). Each sample was then spiked with the exogenous miRNA, cel-mir-39 (final concentration, $2.5 \times 10^{-5} \mathrm{pM}$ ), and total RNA was extracted using the miRNeasy mini kit (Qiagen) as per manufacturer's instructions.

Quantification of mRNA levels in granulosa cell pellets involved a RT step using SuperScript III (Life Technologies Ltd.) and random primers (Promega), followed by quantitative PCR (qPCR) using the SensiFAST SYBR Lo-ROX Kit (Bioline Reagents Ltd., London, UK) on a Mx3005P real-time PCR system (Stratagene, La Jolla, CA, USA). Equine-specific primer sequences for CYP11A1, CYP19A1, PTGS2, LHCGR, PTEN, RASA1, BTG2, MECP2, SMAD4, and 18S (Table 1) that span in each case two different exons within the corresponding genomic sequence were used, and the absence of genomic DNA amplification was confirmed by including a reverse transcriptase negative control. The abundance of each target mRNA was calculated with Mx3000P real-time PCR system analysis software, using the cycle threshold $(C \mathrm{t})$ for each sample relative to $C$ t values obtained from a standard curve constructed from serial dilutions of a pool of equine granulosa cells to which an arbitrary mRNA copy number was assigned. The resulting values for each sample were divided by the corresponding value for $18 \mathrm{~S}$.

Quantification of miRNA levels in granulosa cells and follicular fluid samples was performed using miScript II RT and miScript SYBR Green PCR kits (Qiagen), using commercial primers complementary to equine miRNA and U6B small nuclear RNA sequences and to cel-mir-39. The abundance of each miRNA was calculated from each $C t$ value relative to $\mathrm{Ct}$ values obtained from standard curves constructed from serial dilutions of pools of granulosa cells or follicular fluid samples to which an arbitrary miRNA copy number was assigned, and the corresponding U6B or cel-miR-39 values were used to normalize miRNA expression in granulosa cells and follicular fluid samples respectively. Samples with $C t$ values $>35$ were not used for analyses.

\section{Statistical analyses}

Outlier values were identified from data sets using Dixon's test $(P<0.05)$ and removed before analyses. Analyses were done using SigmaStat (version 4.0). Data sets were tested for normality and homogeneity of variance and were logtransformed if necessary. Data were then subjected to oneway ANOVA. If the overall analysis revealed significant differences, then individual means were compared using Tukey's test. Data sets involving only two groups (Experiment 2) were analyzed using unpaired $t$-tests. By definition, $P<0.05$ indicated statistical significance whereas mean differences involving $P$ values between 0.05 and 0.1 were classified as tending to be significant. All results are shown as mean \pm S.E.M.

\section{Results}

\section{Experiment 1}

Follicular fluid was collected from dominant (DO) and largest subordinate (S) follicles of an ovulatory wave from each of six mares. An actively growing dominant anovulatory (DA) follicle was detected in five of the six mares during mid-cycle and was also aspirated. As expected, at the time of aspiration, DO follicles were larger than $S$ follicles $(34.1 \pm 0.5$ vs $25.3 \pm 0.7 \mathrm{~mm}$ respectively; $P<0.001)$ and of similar $(P=0.22)$ size to DA follicles $(32.7 \pm 1.8 \mathrm{~mm})$. In addition, net growth during $24 \mathrm{~h}$ before aspiration was smaller $(P=0.005)$ for $\mathrm{S}$ follicles $(0.2 \pm 0.8 \mathrm{~mm})$ than for $\mathrm{DO}$ follicles $(3.2 \pm 0.5 \mathrm{~mm})$ but similar $(P=0.15)$ between $\mathrm{DO}$ and DA follicles $(2.2 \pm 0.8 \mathrm{~mm})$. Only a few of the granulosa cell pellets collected from $\mathrm{S}$ and DA follicles provided RNA of sufficient quality for qPCR and therefore they were not used for analyses.

\section{Follicular fluid steroid and IGF1 levels}

The levels of selected markers of follicle maturation (progesterone, estradiol and IGF1) (Donadeu \& Ginther $2002 b$ ) were first determined in follicular fluid (Fig. 1). Relative to DO follicles, follicular fluid levels of progesterone were lower in $S$ follicles $(P<0.01)$ and tended to be lower $(P=0.06)$ in DA follicles, whereas estradiol levels were lower $(P<0.01)$ in $S$ follicles than in either DO or DA follicles. Mean levels of IGF1 were $>40$-fold lower $(P<0.01)$ both of $S$ and DA follicles than in DO follicles.

\section{miRNA levels in follicular fluid}

Changes in the levels of miRNAs putatively involved in follicular cell survival (miR-21 and miR-23a), proliferation (miR-145, miR-503, and miR-224), estradiol

Table 1 Primer sequences used in mRNA analyses.

\begin{tabular}{lll}
\hline Gene & Accession number & Sequence $\left(5^{\prime}-3^{\prime}\right)$ sense/antisense \\
\hline CYP11A1 & NM_001082521.1 & ACAGGCGCATGGAGCAGCAG/TCCCCTGGCGCTCCCCAAAT \\
CYP19A1 & NM_001081805.1 & CGCAAAGCCTTAGAGGATGA/ACCATGGCGATGTACTTTCC \\
PTGS2 & NM_001081775.1 & TGGTCTGGTGCCTGGCCTGA/AGCCGCTCAGGTGCTGTACG \\
LHCGR & XM_001917633.2 & CCCGGTTAAAATACCTAAGC/AGTGTCGTCCCGTGAA \\
PTEN & NM_174324 & TTGCCTGTGTGTGGTGACAT/TCTGAGGTTTCCTCTGGTCCT \\
RASA1 & GM_589440 & GCTTTATCCAGTTGCACCACC/TGTGTAAGGGAGATGGCTCG \\
BTG2 & NM_001034677 & CTTCAGGTTTTCGGCAGAGC/GTAGCCAGAGCCCTTGGATG \\
MECP2 & NM_174817 & CGACGCTCCATCATTCGAGA/AGGTGTCGCCTACCTTTTCG \\
SMAD4 & XR_028231.2 & GACTGGAAGTAGGACCGCAC/TCAGGAGCAGGGTGATTGGA \\
$18 S$ & NM_001192423.1 & GCTGGCACCAGACTTG/GGGGAATCAGGGTTCG \\
\hline
\end{tabular}



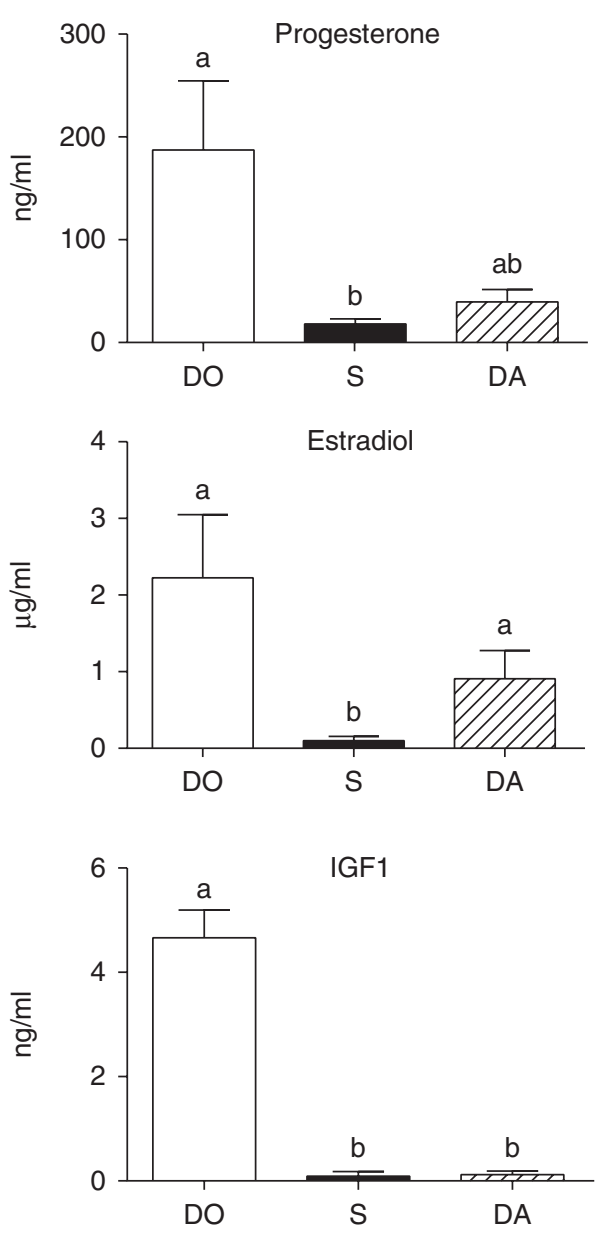

Figure 1 Mean ( \pm S.E.M.) concentrations of progesterone, estradiol, and IGF1 in follicular fluid from dominant ovulatory (DO, white bars), largest subordinate ( $\mathrm{S}$, black bars), and dominant anovulatory (DA, hatched bars) follicles ( $n=6,6$, and 5 mares). There was a main effect of follicle group on each of progesterone $(P=0.002)$, estradiol $(P=0.001)$, and IGF1 $(P<0.001)$. Different superscripts (a and b) indicate differences between group means $(P<0.05)$. For progesterone, DO and DA means tended to be different $(P=0.06)$.

production (miR-224, miR-383, and miR-378), and terminal differentiation (miR-132 and miR-212) were determined in follicular fluid (Fig. 2). Mean levels of miR145 and miR-378 were threefold to sevenfold higher in $S$ follicles than in the other two follicle categories $(P \leq 0.01)$, and there was a tendency $(P=0.06)$ for higher mean levels of miR-132 in S follicles than in DO or DA follicles. However, there were no significant differences between follicle groups in the levels of miR-21, miR-23a, miR-503, and miR-212. miR-224 and miR-383 could not be accurately measured due to low abundance $(\mathrm{Ct}>35)$ in most of the follicle samples analyzed. Similarly, levels of the abundant cell small RNA transcript, U6B, were not detected in follicular fluid samples from either of the two experiments, suggesting that miRNA levels detected in follicular fluid are a product primarily of secretion by follicular cells rather than cell lysis.

\section{Experiment 2}

Once the dominant follicle of an ovulatory wave reached $>32 \mathrm{~mm}$, the follicle was aspirated (DO follicle) or an ovulatory dose of hCG was administered and follicle aspiration was performed $24 \mathrm{~h}$ later (luteinizing follicle, L). Mean diameter of DO follicles at the time of aspiration was $34.5 \pm 0.2 \mathrm{~mm}$. Mean diameters of $L$ follicles at the time of hCG injection and at the time of aspiration $24 \mathrm{~h}$ later were $34.2 \pm 0.5$ and $36.5 \pm 0.6 \mathrm{~mm}$ respectively. One mare developed an ultrasonically detectable hemorrhagic anovulatory follicle (Ginther et al. 2007) after the first follicle aspiration and also failed to ovulate within 3 months after the second follicle aspiration. This mare was deemed to be transitioning into winter anestrus and was removed from analysis.

\section{Steroid, $P G E_{2}$, and $m R N A$ responses to $h C G$ administration}

Levels of different markers associated with ovulation were first determined in follicular fluid to confirm that differences between DO and $\mathrm{L}$ follicles were indeed attributable to the effects of hCG. As shown in Fig. 3, progesterone levels in follicular fluid markedly increased after hCG administration, as indicated by threefold higher levels in $\mathrm{L}$ follicles than in DO follicles $(P=0.05)$, whereas a twofold decrease in mean estradiol levels in $\mathrm{L}$ follicles tended towards significance $(P=0.09)$. An increase in mean $\mathrm{PGE}_{2}$ levels after hCG administration did not reach significance $(P=0.18)$.

To complement these analyses in follicular fluid, mRNA levels of steroid- and PG-synthesizing enzymes as well as $\mathrm{LH}$ receptor were determined in granulosa cell pellets (Fig. 4). Two individual aspirates from DO follicles produced pellets that were either very small or had gross blood contamination and therefore could not be used for analyses. Analyses of the remaining samples revealed a dramatic decrease in the expression of CYP19A1 $(P=0.005)$ together with a tendency $(P=0.07)$ for increased PTGS2 levels after hCG administration. Consistent with these changes, LHCGR levels were reduced $(P=0.005)$ in $\mathrm{L}$ follicles. As expected, mean $18 \mathrm{~S}$ levels were not different $(P>0.2)$ between DO and $\mathrm{L}$ follicles.

\section{miRNA expression responses to $h C G$ administration}

The levels of miRNAs examined in Experiment 1 were also determined in samples collected from DO and $\mathrm{L}$ follicles. Mean follicular fluid levels of miR-132 were ninefold higher $(P=0.01)$ in $\mathrm{L}$ follicles than in $\mathrm{DO}$ follicles, whereas mean levels of miR-21 $(P=0.09)$ and miR-212 $(P=0.08)$ tended to be higher in fluid from $L$ follicles (Fig. 5A). To determine whether follicular fluid levels of miRNAs reflected changes in granulosa cell expression, changes in miRNA expression were also 

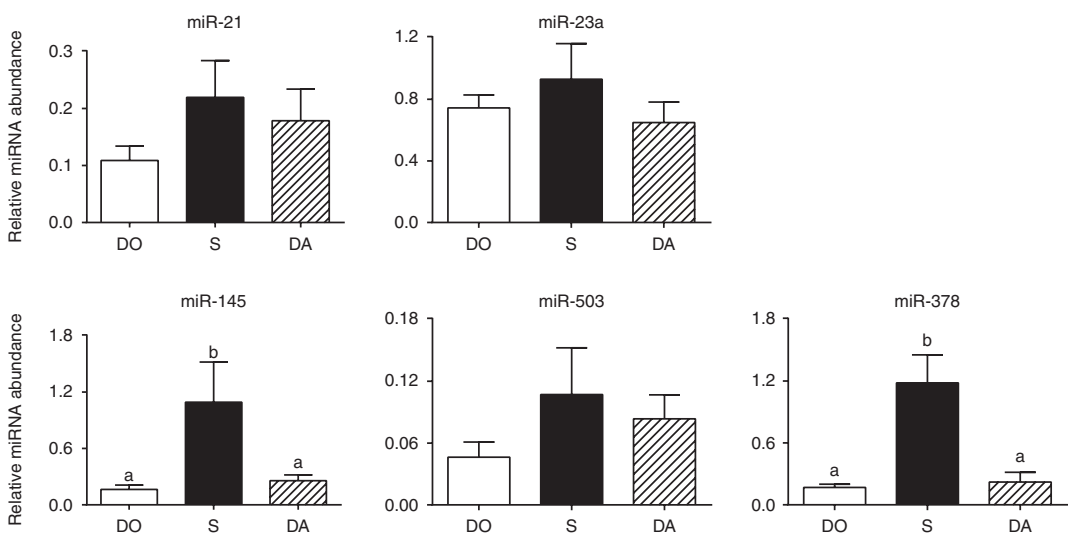
Figure 2 Relative levels (mean \pm S.E.M.) of miRNAs
(normalized to levels of spiked-in cel-miR-39) in
follicular fluid from dominant ovulatory (DO, white
bars), largest subordinate ( $\mathrm{S}$, black bars), and
dominant anovulatory (DA, hatched bars) follicles
( $n=6,6$, and 5 mares). There was a main effect of
follicle group for miR-145 ( $P=0.003)$, miR-378
$(P<0.001)$, and miR-132 ( $P=0.056)$. Different
superscripts (a and b) indicate differences between
group means $(P<0.05)$.
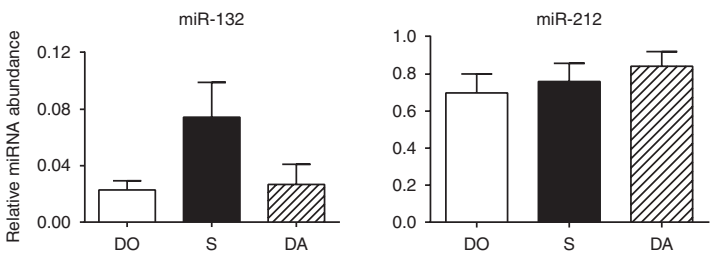

quantified in granulosa cell pellets (Fig. 5B). Consistent with changes in follicular fluid, the levels of miR-21, miR-132, and miR-212 were higher (6-, 18-, and 43-fold respectively; $P<0.05)$ in granulosa cells from $L$ follicles than in those from DO follicles. In contrast, levels of miR-503 tended to be reduced $(P=0.09)$ in $L$ follicles. Although, as in Experiment 1, levels of miR-224 and miR-383 could not be detected in follicular fluid, miR-224 (but not miR-383) was detected in all granulosa cell samples and its levels were fivefold higher $(P=0.02)$ in $\mathrm{L}$ follicles than in DO follicles. Mean granulosa cell levels of $\mathrm{U} 6 \mathrm{~B}$ were similar $(P>0.2)$ in $\mathrm{DO}$ and $\mathrm{L}$ follicles.

\section{Putative follicular targets of miRNAs}

Finally, to gain insight into the potential involvement of miRNAs in ovulation, a list of potential targets of the four miRNAs whose levels increased in L follicles, namely miR-21, miR-224, miR-132, and miR-212, were obtained from miRTarBase (release 3.5), a database of experimentally validated miRNA targets in human, mouse, and rat (Hsu et al. 2011). Putative targets that were common to at least two miRNAs were selected; this yielded four genes, PTEN, BTG2, RASA1, and MECP2, that were simultaneous targets of miR-21 and either or both of miR-132 and miR-212 as candidates for further testing. In addition, we quantified the expression of SMAD4, an experimentally validated miR-224 target in murine granulosa cells (Yao et al. 2010). QPCR analyses of granulosa cell pellets showed that the levels of PTEN $(P=0.004), R A S A 1 \quad(P=0.009)$, and SMAD4 $(P=0.03)$ were lower in $L$ follicles than in DO follicles (Fig. 6) whereas levels of BTG2 were similar $(P=0.3)$ between the two groups of follicles (data not shown). We were unable to detect MECP2 transcript levels in granulosa cell samples. Functional miRNA recognizing elements
(MREs) have been experimentally identified for miR-21 in the 3'UTRs of human PTEN and RASA1 (Lu et al. 2008, Zhang et al. 2010) and for miR-224 in the murine Smad4 sequence (Yao et al. 2010); moreover, a comparison between orthologs revealed conservation of these target sequences in the horse (Fig. 6).

\section{Discussion}

Building on the findings from previous cell culture work, this is the first study, to our knowledge, to examine the association of a list of candidate functional miRNAs with a range of defined stages of follicle development in vivo. In Experiment 1, DO, S, and DA follicles were compared to establish follicular fluid miRNA levels differentially associated with follicle selection (DO vs S) and with maturation leading to ovulatory competence (DO vs DA). We hypothesized that follicle selection would be associated with changes in miRNAs putatively involved in granulosa cell survival (miR-21 and miR23a), proliferation (miR-145, miR-503, and miR-224), and/or estradiol production (miR-224, miR-383, and miR-378). Interestingly, miR-145 and miR-378 were present at significantly higher levels in fluid from subordinate follicles (S) than from healthy growing dominant follicles (DO and DA). miR-145 has antiproliferative effects in different cell types including mouse granulosa cells where this miRNA targets Acvr1 and Ccdn2 (Yan et al. 2012). Although in Experiment 1 it was not possible to analyze the levels of these targets in granulosa cells, reported miR-145 MREs in murine Acvr1 and $C c d n 2$ are highly conserved across species including the horse (Targetscan, release 5.2; data not shown), further suggesting an involvement of this miRNA in follicle regression. 

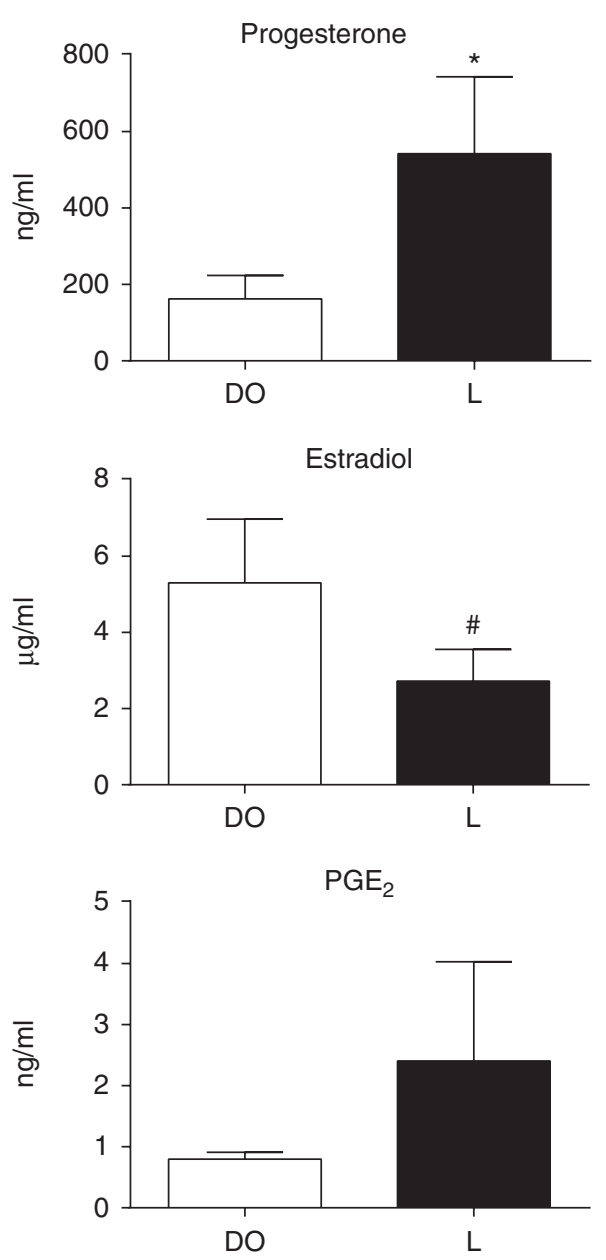

Figure 3 Mean ( \pm S.E.M.) concentrations of progesterone, estradiol, and $\mathrm{PGE}_{2}$ in follicular fluid from dominant ovulatory (DO, white bars) and luteinizing ( $L$, black bars) follicles ( $n=5$ mares/group). Mean differences are indicated by ${ }^{*} P<0.05$ or ${ }^{*} P<0.1$.

The association between high levels of miR-378 and reduced levels of estradiol in subordinate follicles is in agreement with the demonstration that the estradiolsynthesizing enzyme, CYP19A1, is a target of miR-378 in pig granulosa cells in vitro (Xu et al. 2011). In this context, however, a decrease in CYP19A1 levels in granulosa cells following hCG administration in Experiment 2 occurred without concomitant significant changes in miR-378 levels, an observation that does not support a role for this miRNA in regulating CYP19A1 during ovulation. Furthermore, the two reported MREs targeted by miR-378 in the porcine CYP19A 1 are poorly conserved in other species (Xu et al. 2011) including the horse (data not shown). Altogether, and pending experimental validation in other species, the present results do not support a physiological involvement of miR-378 as a core regulator of estradiol production in equine follicles but raise the question of whether miR-378 regulation of CYP19A1 only occurs during specific stages of follicular development, perhaps through different MREs to those reported in the pig. Alternatively, the increase in levels of miR-378 in subordinate follicles may be related to a reported role in mediating cell death in some tissues (Knezevic et al. 2012). In addition to miR-145 and miR-378, mean miR-132 levels tended to be elevated in subordinate follicles, an observation that will need to be confirmed in future studies. Further to its apparent involvement in ovulation (see below), miR-132 plays diverse roles in different body tissues including immune mediation and tissue remodeling (Ucar et al. 2010, Nahid et al. 2013), which may explain the increase in the levels of this miRNA during follicle regression.

Our finding in experiment 1 of reduced mean levels of IGF1 in DA relative to DO follicles and a tendency for progesterone levels to be lower in DA follicles is in agreement with earlier reports on deficient steroidogenesis and IGF1 production by dominant follicles during the luteal phase in mares (Goudet et al. 1999, Bridges et al. 2002) and indicates the developmental deficiencies of DA follicles, presumably induced by reduced circulating LH levels (Schauer et al. 2011). However, despite the differences in IGF1 and progesterone levels, follicular fluid levels of the miRNAs examined were not different between DO and DA follicles, suggesting that these miRNAs are not primarily involved in the LH-driven final maturation of dominant follicles.

In Experiment 2, we shifted our focus to the changes in follicular miRNA profiles induced by an ovulatory dose of hCG (DO vs L follicles). We used a common approach to study temporal gene responses to an ovulatory stimulus in vivo (Boerboom \& Sirois 1998, Fiedler et al. 2008, Gilbert et al. 2011) by determining miRNA levels in two groups of mares before and after hCG administration. An increase in progesterone levels together with a decrease in estradiol, CYP19A1, and LHCGR in $\mathrm{L}$ follicles are consistent with reported changes after induction of ovulation (Boerboom \& Sirois 1998, Belin et al. 2000). L follicles were collected $24 \mathrm{~h}$ after hCG administration, before the expected time of follicular rupture (Gastal et al. 2006). The mean increase in $\mathrm{PGE}_{2}$ levels was not significant in these follicles whereas PTGS2 levels tended to be higher than in DO follicles; these findings are consistent with the demonstration that follicular PTGS2 is significantly induced in the mare at a mean of $30 \mathrm{~h}$ following an ovulatory dose of hCG (Boerboom \& Sirois 1998).

Unlike $S$ and DA follicles in Experiment 1, aspirates from $D O$ and $L$ follicles yielded cell pellets that were large enough and of sufficient quality for qPCR analyses, providing the opportunity to determine gene expression levels in granulosa cells. There was a good agreement in miRNA profiles between follicular fluid and granulosa cells, with marked increases in granulosa cell levels of miR-21, miR-132, and miR-212 in L follicles being reflected in increases, albeit of smaller magnitude, in the follicular fluid levels of these miRNAs. Ct values obtained 

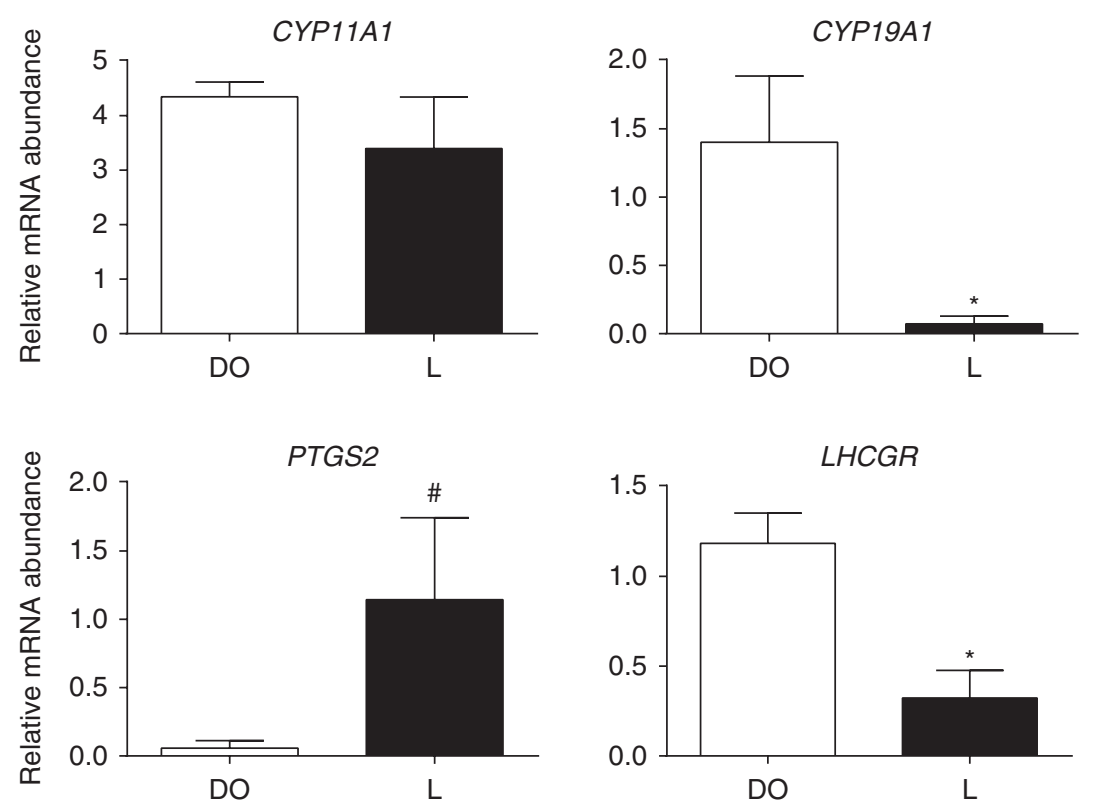

Figure 4 Relative mRNA levels (mean \pm S.E.M.) of
CYP11A1, CYP19A1, PTGS2, and LHCGR
(normalized to $18 \mathrm{~S}$ levels) in granulosa cell pellets
collected from dominant ovulatory (DO, white
bars) and luteinizing (L, black bars) follicle aspirates
( $n=5$ mares/group). Mean differences are indicated
by ${ }^{*} P<0.05$ or ${ }^{*} P<0.1$.

for miR-224 in granulosa cells (between 30 and 35 in all cases) were, in general, higher than the values obtained for other miRNAs; this is consistent with our previous finding of a relatively low abundance of miR-224 sequences in sheep follicles (McBride et al. 2012) and may explain our inability to detect miR-224 in follicular fluid in this study. Also, consistent with the present results, we did not detect the sequence for miR-383 in sheep follicular tissues (McBride et al. 2012). Taken together, these observations support the conclusion that the follicular granulosa cell compartment is a major contributor to miRNA levels in follicular fluid (da Silveira et al. 2012).

An earlier study reported the upregulation of three miRNAs, miR-21, miR-132, and miR-212, in granulosa cells in response to an ovulatory dose of hCG in mice (Fiedler et al. 2008). In that context, the present results on mares are important as they indicate a conserved involvement of this miRNA triad in mammalian ovulation. Carletti et al. (2010) showed that in mice the increase in miR-21 during ovulation acted to promote granulosa cell survival; however, they failed to identify candidate gene targets involved in this effect. In addition, the role of miR-132 and miR-212 (two miRNAs originating from a chromosome 11 cluster in the horse and with a shared seed sequence) in follicular cells is not known (Fiedler et al. 2008). To clarify the functional involvement of these miRNAs during ovulation, we sought to identify candidate gene targets in equine follicles. Our results support the notion that PTEN and RASA1, two cell signaling regulators, are targeted by the combination of miR-21, miR-132, and miR-212 as indicated by the following: i) the expression of PTEN and RASA1 clearly decreased in equine preovulatory follicles simultaneously with an increase in the levels of miR-21, miR-132, and miR-212 in response to hCG (Figs 5 and 6), ii) PTEN and RASA1 are

bonafide targets of these three miRNAs in humans and rodents (miRTarBase, release 3.5), and iii) MREs involved in these interactions are well conserved in the horse (Fig. 6). In agreement with these findings, knockdown of miR-21 in mouse granulosa cells resulted in a transient increase in PTEN protein levels (Carletti et al. 2010). A decrease in the expression of PTEN and RASA1 during ovulation is consistent with previous follicular data on cattle (Rao et al. 2011) and with the involvement of these two gene products in negative regulation of AKT and RAS, two cell signaling pathways involved in survival and proliferation/differentiation, and which activation in granulosa cells is central to $\mathrm{LH}$-induced oocyte maturation, ovulation, and luteinization (Gilbert et al. 2011, Fan et al. 2012). In contrast to PTEN and RASA1, we did not detect any differences in transcript levels of the antiproliferative factor, BTG2, between DO and $L$ follicles. BTG2 is a bonafide target of miR-21 and miR-132 in mouse and human cells respectively (Alvarez-Saavedra et al. 2011, Liu et al. 2011). Although only limited amounts of granulosa cells could be collected from individual follicles in live mares, which precluded analyses of protein levels in these samples, we cannot exclude a potential miRNA effect on levels of BTG2 protein. Furthermore, in this study, a single time-point after hCG administration ( $24 \mathrm{~h}$ ) was considered, which prevents any conclusion on whether the changes observed in putative target levels were indeed causally related to changes in miRNAs; further and more detailed examination of gene expression changes following an ovulatory stimulus, possibly involving in vitro approaches, should be performed to clarify the present results. Nonetheless, and taking into account the above caveats, the present findings expand significantly on previous results on mice by providing substantial evidence of a combined effect of the miRNA triad, 


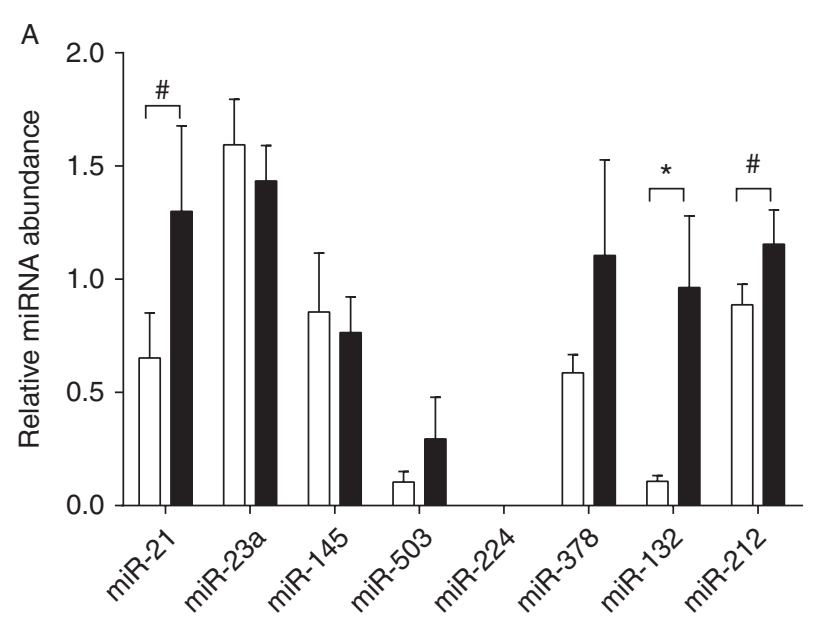

miR-224 (Yao et al. 2010). In this study, relative to DO follicles, L follicles had simultaneously elevated levels of miR-224 and reduced levels of SMAD4; this, together with the fact that a miR-224 MRE in the mouse SMAD

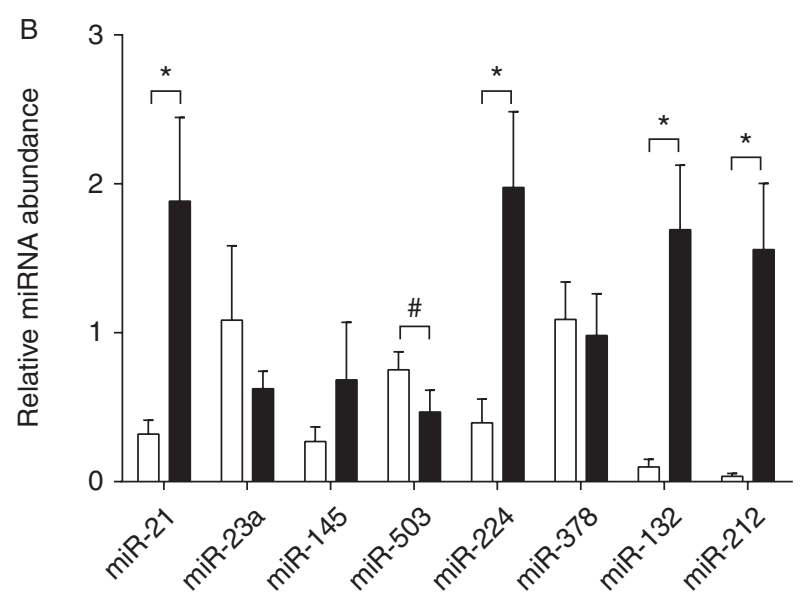

Figure 5 Relative miRNA levels (mean \pm S.E.M.) in (A) follicular fluid (normalized to spiked-in cel-miR-39 levels) and (B) granulosa cells (normalized to U6B levels) collected from dominant ovulatory (DO, white bars) and luteinizing ( $\mathrm{L}$, black bars) follicles ( $n=5$ mares/group). Within each set and for each miRNA, differences between DO and $\mathrm{L}$ means are indicated by ${ }^{*} P<0.05$ or ${ }^{\#} P<0.1$.

miR-21, miR-132, and miR-212, in physiologically regulating follicular cell survival and differentiation during ovulation in the mare.

Considering our results in Experiment 2 together with the reported role of miR-21 as a pro-survival factor in mouse preovulatory follicles (Carletti et al. 2010), the finding in Experiment 1 that miR-21 levels were similar between subordinate and dominant follicles, rather than reduced in subordinate follicles, was not expected. This finding, however, can be interpreted in light of the different cellular roles attributed to miR-21, in addition to its best known function as an anti-apoptotic factor, including an involvement in mediating immune and fibrotic responses (Kumarswamy etal. 2011), which reportedly play an active role in follicle atresia (Suzuki et al. 1998).

There is little information on the effects of miR-224 in non-transformed cells of any species, and this includes a report in granulosa cells from mouse preantral follicles in which SMAD4 was identified as a bonafide target of
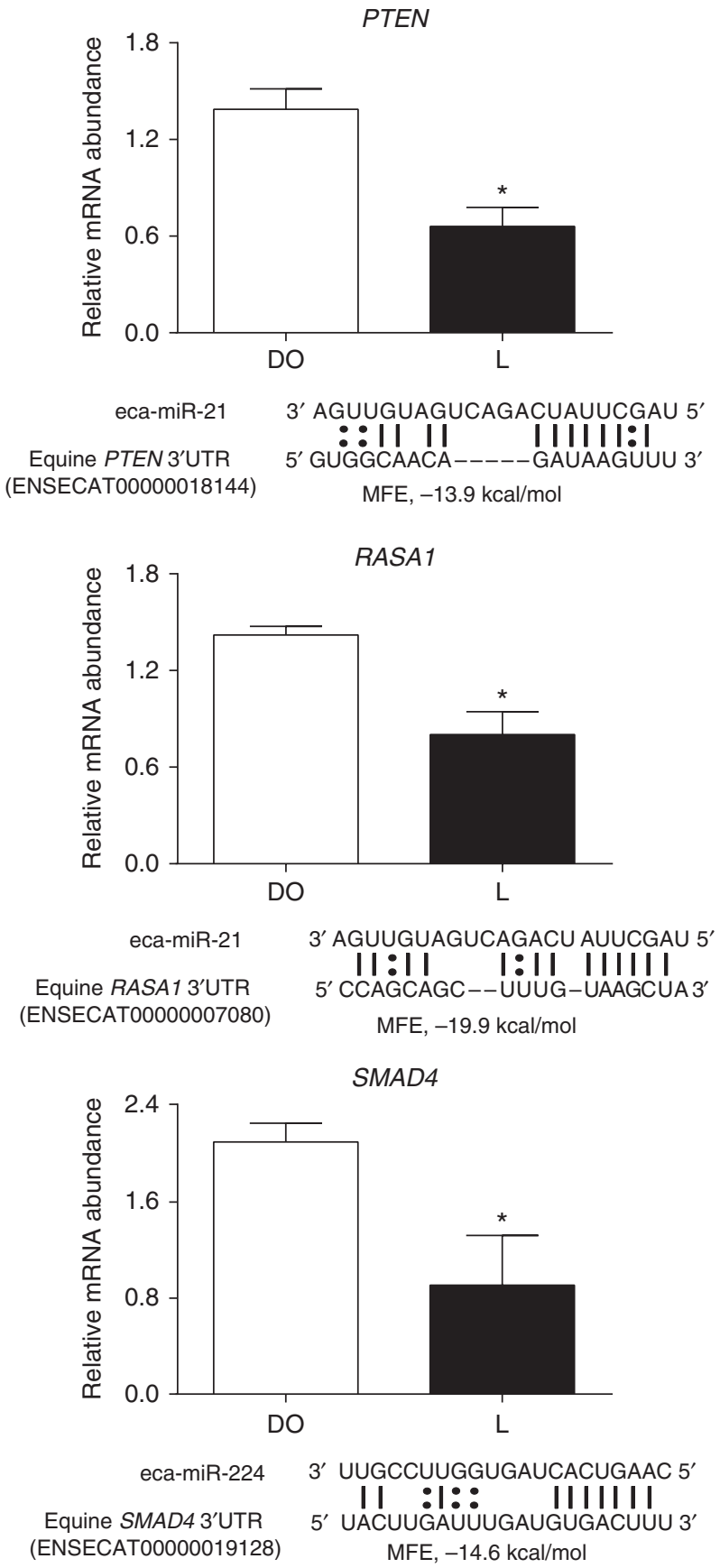

Figure 6 Relative mRNA levels (mean \pm s.E.M.) of PTEN, RASA1, and SMAD4 (normalized to $18 \mathrm{~S}$ levels) in granulosa cell pellets collected from dominant ovulatory (DO, white bars) and luteinizing (L, black bars) follicle aspirates ( $n=5$ mares/group). Mean differences are indicated by $* P<0.05$. Putative interactions between equine $3^{\prime}$ UTR sequences and miRNAs (based on experimentally validated target sites in conserved human and mouse orthologs) were produced using the RNAhybrid tool (Rehmsmeier et al. 2004) and are shown for each target. MFE, minimum free energy. 
$3^{\prime}$ UTR is fully conserved in the horse (Fig. 6), supports an effect of miR-224 in suppressing SMAD4 during ovulation. Interestingly, the demonstration that an ovarian deficiency in SMAD4 is associated with premature luteinization in mice (Pangas et al. 2006) raises the possibility of a novel physiological role of miR-224 in terminal differentiation of follicular cells, a hypothesis that will need further testing.

Finally, a tendency for a decrease in granulosa cell levels of miR-503 following administration of an ovulatory dose of hCG in this study is in line with previous results from mice (Lei et al. 2010) and sheep (McBride et al. 2012) and with data indicating that miR-503 can regulate the expression of different genes associated with proliferation and differentiation in granulosa cells (Lei et al. 2010).

In summary, building on data from previous in vitro studies, this study in mares examined the physiological association of a subset of candidate miRNAs with different stages of follicle development representing selection, maturation, and ovulation. The results show a differential increase in the levels of miR-145, miR-378, and miR-132 in the follicular fluid of subordinate follicles relative to dominant follicles and an increase in follicular levels of miR-21, miR-132, miR-212, and miR-224 following administration of an ovulatory dose of hCG. Furthermore, we provide novel evidence suggesting an involvement of these miRNAs in regulating cell survival and differentiation through targeting of PTEN, RASA1, and SMAD4 during ovulation. In addition, the present results indicate that follicular fluid may be useful in monitoring follicular cell levels of miRNAs.

\section{Declaration of interest}

The authors declare that there is no conflict of interest that could be perceived as prejudicing the impartiality of the research reported.

\section{Funding}

This work was supported by Institute Strategic Grant Funding from the Biotechnology and Biological Sciences Research Council.

\section{Acknowledgements}

The authors are grateful to the staff at Dryden Farm, Roslin Institute, for animal care.

\section{References}

Alvarez-Saavedra M, Antoun G, Yanagiya A, Oliva-Hernandez R, Cornejo-Palma D, Perez-Iratxeta C, Sonenberg N \& Cheng H-YM 2011 miRNA-132 orchestrates chromatin remodeling and translational control of the circadian clock. Human Molecular Genetics 20 731-751. (doi:10.1093/hmg/ddq519)
Belin F, Goudet G, Duchamp G \& Gérard N 2000 Intrafollicular concentrations of steroids and steroidogenic enzymes in relation to follicular development in the mare. Biology of Reproduction 62 1335-1343. (doi:10.1095/biolreprod62.5.1335)

Boerboom D \& Sirois J 1998 Molecular characterization of equine prostaglandin $\mathrm{G} / \mathrm{H}$ synthase-2 and regulation of its messenger ribonucleic acid in preovulatory follicles. Endocrinology 139 1662-1670. (doi:10.1210/en.139.4.1662)

Bridges TS, Davidson TR, Chamberlain CS, Geisert RD \& Spicer LJ 2002 Changes in follicular fluid steroids, insulin-like growth factors (IGF) and IGF-binding protein concentration, and proteolytic activity during equine follicular development. Journal of Animal Science $\mathbf{8 0}$ 179-190.

Carletti MZ, Fiedler SD \& Christenson LK 2010 MicroRNA 21 blocks apoptosis in mouse periovulatory granulosa cells. Biology of Reproduction 83 286-295. (doi:10.1095/biolreprod.109.081448)

Carnevale EM 2008 The mare model for follicular maturation and reproductive aging in the woman. Theriogenology 69 23-30. (doi:10.1016/j.theriogenology.2007.09.011)

Donadeu F \& Ginther OJ 2002a Follicular waves and circulating concentrations of gonadotrophins, inhibin and oestradiol during the anovulatory season in mares. Reproduction 124 875-885. (doi:10.1530/rep.0.1240875)

Donadeu FX \& Ginther OJ 2002 $b$ Changes in concentrations of follicular fluid factors during follicle selection in mares. Biology of Reproduction 66 1111-1118. (doi:10.1095/biolreprod66.4.1111)

Donadeu FX \& Pedersen HG 2008 Follicle development in mares. Reproduction in Domestic Animals 43 (Suppl 2) 224-231. (doi:10.1111/j.1439-0531.2008.01166.x)

Donadeu FX, Schauer SN \& Sontakke SD 2012 Involvement of miRNAs in ovarian follicular and luteal development. Journal of Endocrinology 215 323-334. (doi:10.1530/JOE-12-0252)

Doyle LK, Hogg CO, Watson ED \& Donadeu FX 2008 Seasonal effects on the response of ovarian follicles to IGF1 in mares. Reproduction $\mathbf{1 3 6}$ 589-598. (doi:10.1530/REP-07-0507)

Fan H-Y, Liu Z, Mullany LK \& Richards JS 2012 Consequences of RAS and MAPK activation in the ovary: the good, the bad and the ugly. Molecular and Cellular Endocrinology 356 74-79. (doi:10.1016/j.mce. 2011.12.005)

Fiedler SD, Carletti MZ, Hong X \& Christenson LK 2008 Hormonal regulation of microRNA expression in periovulatory mouse mural granulosa cells. Biology of Reproduction 79 1030-1037. (doi:10.1095/ biolreprod.108.069690)

Gastal EL, Silva LA, Gastal MO \& Evans MJ 2006 Effect of different doses of hCG on diameter of the preovulatory follicle and interval to ovulation in mares. Animal Reproduction Science 94 186-190. (doi:10.1016/j. anireprosci.2006.04.007)

Gilbert I, Robert C, Dieleman S, Blondin P \& Sirard MA 2011 Transcriptional effect of the LH surge in bovine granulosa cells during the peri-ovulation period. Reproduction 141 193-205. (doi:10.1530/ REP-10-0381)

Ginther OJ, Gastal EL, Gastal MO, Bergfelt DR, Baerwald AR \& Pierson RA 2004 Comparative study of the dynamics of follicular waves in mares and women. Biology of Reproduction 71 1195-1201. (doi:10.1095/biolreprod.104.031054)

Ginther OJ, Beg MA, Gastal EL, Gastal MO, Baerwald AR \& Pierson RA 2005 Systemic concentrations of hormones during the development of follicular waves in mares and women: a comparative study. Reproduction 130 379-388. (doi:10.1530/rep.1.00757)

Ginther OJ, Gastal EL, Gastal MO \& Beg MA 2007 Incidence, endocrinology, vascularity, and morphology of hemorrhagic anovulatory follicles in mares. Journal of Equine Veterinary Science 27 130-139. (doi:10.1016/j.jevs.2007.01.009)

Goudet G, Belin F, Bézard J \& Gérard N 1999 Intrafollicular content of luteinizing hormone receptor, $\boldsymbol{\alpha}$-inhibin, and aromatase in relation to follicular growth, estrous cycle stage, and oocyte competence for in vitro maturation in the mare. Biology of Reproduction 60 1120-1127. (doi:10.1095/biolreprod60.5.1120)

Hossain MM, Ghanem N, Hoelker M, Rings F, Phatsara C, Tholen E, Schellander K \& Tesfaye D 2009 Identification and characterization of miRNAs expressed in the bovine ovary. BMC Genomics 10443. (doi:10.1186/1471-2164-10-443) 
Hsu S-D, Lin F-M, Wu W-Y, Liang C, Huang W-C, Chan W-L, Tsai W-T, Chen G-Z, Lee C-J, Chiu C-M et al. 2011 miRTarBase: a database curates experimentally validated microRNA-target interactions. Nucleic Acids Research 39 D163-D169. (doi:10.1093/nar/gkq1107)

Knezevic I, Patel A, Sundaresan NR, Gupta MP, Solaro RJ, Nagalingam RS \& Gupta M 2012 A novel cardiomyocyte-enriched microRNA, miR-378, targets insulin-like growth factor 1 receptor: implications in postnatal cardiac remodeling and cell survival. Journal of Biological Chemistry 287 12913-12926. (doi:10.1074/jbc.M111.331751)

Kumarswamy R, Volkmann I \& Thum T 2011 Regulation and function of miRNA-21 in health and disease. RNA Biology 8 706-713. (doi:10.4161/ rna.8.5.16154)

Lei L, Jin S, Gonzalez G, Behringer RR \& Woodruff TK 2010 The regulatory role of Dicer in folliculogenesis in mice. Molecular and Cellular Endocrinology 315 63-73. (doi:10.1016/j.mce.2009.09.021)

Li M, Liu Y, Wang T, Guan J, Luo Z, Chen H, Wang X, Chen L, Ma J, Mu Z et al. 2011 Repertoire of porcine microRNAs in adult ovary and testis by deep sequencing. International Journal of Biological Sciences 7 1045-1055. (doi:10.7150/ijbs.7.1045)

Lin F, Li R, Pan Zx, Zhou B, Yu Db, Wang Xg, Ma Xs, Han J, Shen M \& Liu HI 2012 miR-26b promotes granulosa cell apoptosis by targeting ATM during follicular atresia in porcine ovary. PLOS ONE 7 e38640. (doi:10.1371/journal.pone.0038640)

Liu LZ, Li C, Chen Q, Jing Y, Carpenter R, Jiang Y, Kung HF, Lai L \& Jiang BH $2011 \mathrm{MiR}-21$ induced angiogenesis through AKT and ERK activation and HIF-1 $\alpha$ expression. PLoS ONE 6 e19139. (doi:10.1371/journal.pone. 0019139)

Lu Z, Liu M, Stribinskis V, Klinge CM, Ramos KS, Colburn NH \& Li Y 2008 MicroRNA-21 promotes cell transformation by targeting the programmed cell death 4 gene. Oncogene 27 4373-4379. (doi:10.1038/ onc.2008.72)

McBride D, Carre W, Sontakke SD, Hogg CO, Law A, Donadeu FX \& Clinton M 2012 Identification of miRNAs associated with the follicularluteal transition in the ruminant ovary. Reproduction 144 221-233. (doi:10.1530/REP-12-0025)

Mishima T, Takizawa T, Luo SS, Ishibashi O, Kawahigashi Y, Mizuguchi Y, Ishikawa T, Mori M, Kanda T, Goto T et al. 2008 MicroRNA (miRNA) cloning analysis reveals sex differences in miRNA expression profiles between adult mouse testis and ovary. Reproduction 136 811-822. (doi:10.1530/REP-08-0349)

Nahid MA, Yao B, Dominguez-Gutierrez PR, Kesavalu L, Satoh M \& Chan EK 2013 Regulation of TLR2-mediated tolerance and crosstolerance through IRAK4 modulation by miR-132 and miR-212. Journal of Immunology 190 1250-1263. (doi:10.4049/jimmunol. 1103060)

Pangas SA, Li X, Robertson EJ \& Matzuk MM 2006 Premature luteinization and cumulus cell defects in ovarian-specific Smad4 knockout mice. Molecular Endocrinology 20 1406-1422. (doi:10.1210/me.2005-0462)

Rao JU, Shah KB, Puttaiah J \& Rudraiah M 2011 Gene expression profiling of preovulatory follicle in the buffalo cow: effects of increased IGF-I concentration on periovulatory events. PLOS ONE 6 e20754. (doi:10.1371/journal.pone.0020754)

Rehmsmeier M, Steffen P, Höchsmann M \& Giegerich R 2004 Fast and effective prediction of microRNA/target duplexes. RNA 10 1507-1517. (doi:10.1261/rna.5248604)
Sayed D \& Abdellatif M 2011 MicroRNAs in development and disease. Physiological Reviews 91 827-887. (doi:10.1152/physrev.00006.2010) Schauer SN, Briant C, Ottogalli M, Decourt C, Handel IG \& Donadeu FX 2011 Supplementation of equine early spring transitional follicles with luteinizing hormone stimulates follicle growth but does not restore steroidogenic activity. Theriogenology 75 1076-1084. (doi:10.1016/ j.theriogenology.2010.11.016)

Schauer SN, Guillaume D, Decourt C, Watson ED, Briant C \& Donadeu FX 2013 Effect of luteinizing hormone overstimulation on equine follicle maturation. Theriogenology 79 409-416. (doi:10.1016/j.theriogenology. 2012.08.017)

da Silveira JC, Veeramachaneni DNR, Winger QA, Carnevale EM \& Bouma GJ 2012 Cell-secreted vesicles in equine ovarian follicular fluid contain miRNAs and proteins: a possible new form of cell communication within the ovarian follicle. Biology of Reproduction 8671. (doi:10.1095/biolreprod.111.093252)

Suzuki T, Sasano H, Takaya R, Fukaya T, Yajima A, Date F \& Nagura H 1998 Leukocytes in normal-cycling human ovaries: immunohistochemical distribution and characterization. Human Reproduction 13 2186-2191. (doi:10.1093/humrep/13.8.2186)

Ucar A, Vafaizadeh V, Jarry H, Fiedler J, Klemmt PAB, Thum T, Groner B \& Chowdhury K 2010 miR-212 and miR-132 are required for epithelial stromal interactions necessary for mouse mammary gland development. Nature Genetics 42 1101-1108. (doi:10.1038/ng.709)

Xu S, Linher-Melville K, Yang BB, Wu D \& Li J 2011 Micro-RNA378 (miR-378) regulates ovarian estradiol production by targeting aromatase. Endocrinology 152 3941-3951. (doi:10.1210/en.2011-1147)

Yan G, Zhang L, Fang T, Zhang Q, Wu S, Jiang Y, Sun H \& Hu Y 2012 MicroRNA-145 suppresses mouse granulosa cell proliferation by targeting activin receptor IB. FEBS Letters $586 \quad 3263-3270$. (doi:10.1016/j.febslet.2012.06.048)

Yang X, Zhou Y, Peng S, Wu L, Lin H-Y, Wang S \& Wang H 2012 Differentially expressed plasma microRNAs in premature ovarian failure patients and the potential regulatory function of mir-23a in granulosa cell apoptosis. Reproduction 144 235-244. (doi:10.1530/REP-11-0371)

Yao G, Yin M, Lian J, Tian H, Liu L, Li X \& Sun F 2010 MicroRNA-224 is involved in transforming growth factor- $\beta$-mediated mouse granulosa cell proliferation and granulosa cell function by targeting Smad4. Molecular Endocrinology 24 540-551. (doi:10.1210/me.2009-0432)

Yin M, Lu M, Yao G, Tian H, Lian J, Liu L, Liang M, Wang Y \& Sun F 2012 Transactivation of microRNA-383 by steroidogenic factor- 1 promotes estradiol release from mouse ovarian granulosa cells by targeting RBMS1. Molecular Endocrinology 26 1129-1143. (doi:10.1210/me. 2011-1341)

Zhang JG, Wang JJ, Zhao F, Liu Q, Jiang K \& Yang GH 2010 MicroRNA-21 (miR-21) represses tumor suppressor PTEN and promotes growth and invasion in non-small cell lung cancer (NSCLC). Clinica Chimica Acta 411 846-852. (doi:10.1016/j.cca.2010.02.074)

Received 19 March 2013

First decision 29 April 2013

Revised manuscript received 20 May 2013

Accepted 17 June 2013 